
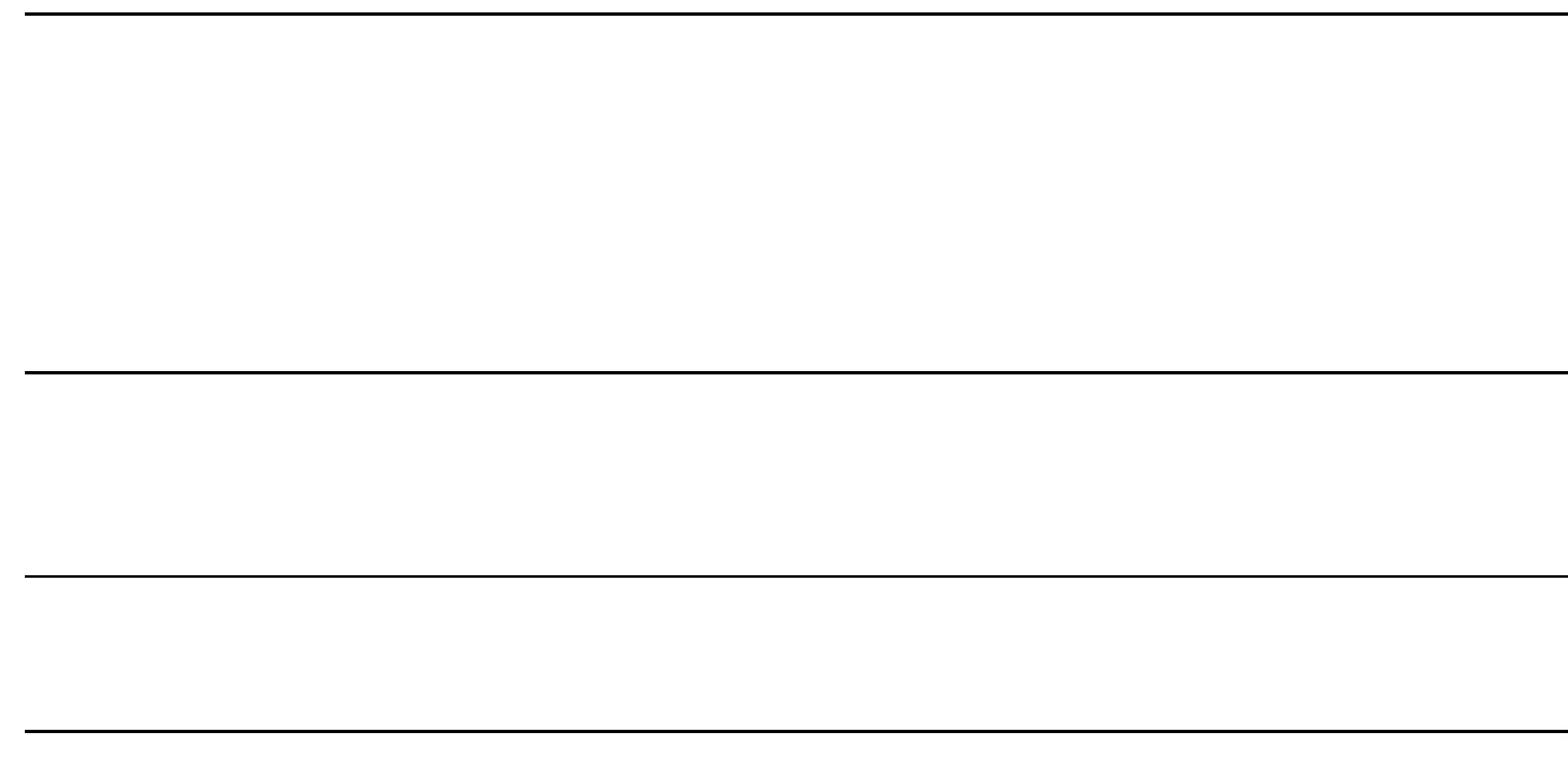


\title{
Silver birch ability to refill fully embolised xylem conduits under tension
}

\author{
Y. Salmon ${ }^{1,2, a \cdot A}$ A. Lintunen ${ }^{1,2} \cdot$ L. Lindfors ${ }^{2,3} \cdot$ H. Suhonen ${ }^{1} \cdot$ S. Sevanto ${ }^{4} \cdot$ T. Vesala ${ }^{1}$ and T. Hölttä ${ }^{2}$ \\ 1 Institute for Atmospheric and Earth System Research Physics, Faculty of Science - University of Helsinki, Finland; \\ 2Institute for Atmospheric and Earth System Research Forest Sciences, Faculty of Agriculture and Forestry, \\ University of Helsinki, Finland; 3Umeå Plant Science Centre, Department of Forest Genetics and Plant Physiology, \\ Swedish University of Agricultural Sciences, Umeå, Sweden; ${ }^{4}$ Earth and Environmental Sciences Division, Los \\ Alamos National Laboratory, Los Alamos, New Mexico, USA.
}

\begin{abstract}
Whether trees have the ability to refill embolised xylem conduits and possibly to do it under tension, i.e. inÉ physiologically active state, has beenÉ topic of research and debate for many years. However, direct experimental evidence for embolism refilling remains scarce. Combining traditional tree hydraulic methods (e.g. hydraulic conductance measurements on excised stems), new approaches (cut end ofÉ branch placed in contact with water held in an agar matrix), and newer technology (X-ray microtomography), we tested the potential of silver birch branches to refill embolised xylem conduits even under negative water potential. The hydraulic method suggests that refilling of embolised xylem can occur even under negative pressure. The successful ability of birch to refill xylem was confirmed by X-ray microtomography. Furthermore, our results suggest that refilling is unlikely to be driven osmotically given the small difference measured between sap and the osmolality of the source water (tap water, agar). Finally, our results have direct influence on the method of choice when studying the vulnerability of silver birch to cavitation.
\end{abstract}

Key words, sapwood, refilling, embolism, methodology, X-ray microtomography, hydraulic, Betula pendula

\section{INTRODUCTION}

tree's ability to refill embolised xylem conduits isÉ central question in predicting how they will be able to cope with drought. However, the issue remains highly controversial and while several mechanisms have been proposed to explain potential xylem refilling (Nardini et al., 2011), experimental evidence remains scarce, possibly due to the lack of widespread affordable methods. X-ray microtomography has been used to study embolism and refilling in excised branches and small saplings (e.g., Cochard et al., 2015). However, this method is not easily available, it is costly and limited to small tissues/ trees.

Here we useÉ simple method relying on traditional hydraulic measurements to test for silver birch's ability to refill while under small tension, i.e., comparable to whatÉ tree might encounter at night. The ability of birch to refill winter-embolised xylem in boreal conditions by creating positive pressure in the xylem during the sap season, i.e. the period in the springtime between soil thawing and bud burst, has been known forÉ long time (e.g., Merwin and Lyon, 1909; Westhoff et al., 2008). Refilling during sap season has been proposed to rely on root pressure and the presence of osmotically active compounds released in the xylem conduits (e.g., Merwin and Lyon, 1909; Westhoff et al., 2008). However, it is not known if birch can also refill fully embolised xylem conduits outside the sap season under tension and if the refilling can happen without root pressure. We hypothesize that: 1) birch branches can refill outside the sap season and without root pressure since earlier work on birch refilling during the sap season showed that hydrostatic pressure aboveground was playingÉ role in refilling (Westhoff et al., 2008); and 2) that the refilling is osmotically driven, since it has been

\footnotetext{
a E-mail: yann.salmon@ helsinki.fi
} 
hypothesized to be the force allowing for refilling under tension (Holbrook and Zwieniecki, 1999; Westhoff et al., 2008).

\section{MATERIALS AND METHODS}

To test whether birch branches can refill we conducted two hydraulic experiments: 1) refilling of fully embolized branches under mild positive pressure, and 2) refilling of fully embolized branches under mild tension. To test whether osmotic mechanisms were involved, we used fresh branches and branches where living cells were killed with heating treatment, and measured osmolality of the xylem sap after refilling. To verify our findings of refilling,É new set of samples was imaged with x-ray microtomography during refilling in the absence of positive or negative pressure.

\section{Plant material}

Silver Birch (Betula pendula Roth.) branches were sampled on randomly selected trees (number of replicate per measurements is given below) on Viikki and Kumpula campuses of the University of Helsinki (Helsinki, Finland) during winter but outside of the sap season.

The sampled branches were one centimetre in diameter and one meter in length at minimum. segment of each branch was recut according to experimental need (see below). Branch water potential 2 was measured with É pressure chamber (PMS-1000, PMS instruments, Albany, OR, USA) until they could be considered fully embolised and nonconductive, i.e. when their water potential was lower than -3MPa. Silver birch P90 (water potential at which $90 \%$ of the hydraulic conductance is lost) is $-2.75 \mathrm{MPa}$ (Herbette and Cochard, 2010).

To control if the refilling is an active process or could be achieved simply by capillary movement within the conducting elements, we sampled additional branches from the same trees and killed the living cells in the xylem prior to measurements. Freshly sampled branches were wrapped in alimentary cling film to avoid desiccation that could trigger cracks in the wood and placed inÉ drying oven at $65^{\circ} \mathrm{C}$ for 24 ä to kill living cells (Stiller and Sperry, 1999). This method was preferred to microwaving the sample, as earlier trials had shown that microwaving was creating cracks in the wood. Furthermore, the heating did not dry the samples because they were covered with É film to prevent dehydration. An earlier study (Lintunen et al., 2017) demonstrated that using the same protocol, the measured water potential was not decreased by the heating.

\section{Refilling under mild positive pressure}

Hydraulic conductance was measured gravimetrically according to Sperry et al. (1988) on both fully embolised fresh branches and killed branches. Briefly, the branches were vertically attached toÉ laboratory stand,É water reservoir was connected with tubing to the top of the branches, creating an overhead pressure of 0.08 Bar. Water flow through the branches was measured withE balance. To ensure that water had to flow through the xylem during the measurements, phloem was peeled off at the point of contact between the branch and the tubing and PTFE tape was used to prevent leaks and direct contact of water with the phloem. If conductance was restored during the measurement inÉ reasonable time period, i.e. withinÉ few hours, we interpreted this as embolism refilling occurring. We choseÉ limit of 12 hours for refilling, because given tens or hundreds of hours, any branches, even the dead ones, would refill passively by capillary forces when placed in contact with water (see Yang and Tyree 1992). Therefore, we expected that no refilling would take place in dead branches over suchÉ short period. However, refilling of living branches during that period would show that refilling is an active process. The maximum vessel length in silver birch has been found to be approximately $16 \mathrm{~cm}$ (Cochard et al., 2010), while the average vessel length is less than wmm (Piispanen et al., 2003). To avoid the artefacts in hydraulic conductance measurements caused by cut-open vessels, $\mathrm{u}$ samples of at least $30 \mathrm{~cm}$ were used. 


\section{Refilling under tension}

To create water tension for refilling, É 10 â $\mathrm{L}^{-1}$ agar (VWR chemicals) solution was created and it's osmolality was measured at $0.04 \mathrm{osmo} / \mathrm{kg}$ withÉ freezing point osmometer (Osmomat 030 cryoscopic osmometer, Gonotec, Berlin, DE). Once the solution had cooled down enough to avoid damaging the living cells, but before the agar solution solidified, we inserted the bottom ofÉ twig to the solution vertically. The twigs were bench-dried to water potentials lower than -3.5 MPa. The living bark was removed toE few $\mathrm{mm}$ above the insertion. Thus, only the xylem was in contact with the agar. The twig was held in place withÉ holder. The top of the twig was left in contact with the ambient environment. The agar solution createdÉ matrix holding the water atÉ water potential of approximately $-0.1 \mathrm{MPa}$ (based on its osmolality and in agreement with the experimental results shown by Ghashghaie et al. (1991) for water potential of agar solution), E value close to what birches would experience at night in boreal regions (Sellin and Kupper, 2005). This value is sufficient to excludeÉ refilling by capillary forces. Based on the Laplace equation $2 \pm \mathrm{tI} / \mathrm{r}$ where 2 is the water potential of the agar solution,I is the surface tension of water $(\sim 0.07 \mathrm{~N} / \mathrm{m})$ andî is vessel radius, which is assumed to be of the order of $10 \mu \mathrm{m}$ (Lintunen et al., 2010)), the water potential at which capillary forces cannot contribute to refilling anymore is of the order of magnitude -0.01 MPa in birch. Thus, refilling in these twigs had to take place against the negative water potential of the matrix and gravity. After 12 hours, the twig water potential and hydraulic conductivity of the sample placed in agar were measured (see above).

\section{Xylem sap osmolality}

The first droplets of the xylem sap from the twig were collected from the "hydraulic conductance" and "refilling under tension" experiments so that osmolality could be measured withÉ freezing point osmometer (Osmomat 030 cryoscopic osmometer). The osmolality of the xylem sap could potentially give information on the refilling mechanism. high osmolality would be expected if refilling occurred by the so called "osmotic refilling mechanism", where solutes would be secreted by the living cells to the refilling vessels thus drawing water for refilling osmotically (Nardini et al., 2011; Tyree et al., 1999).

\section{X-ray microtomography}

To verify refilling of conduits,É full x-ray microtomography scan was conducted onÉ living fully-embolised non-conducting sample $2 ¥-4 \mathrm{MPa}$ ) and É fully-embolised nonconducting killed sample similarly to those used in the two refilling experiments described above. To avoid losing resolution by increasing the field of view, the samples were selected to be smaller in diameter than for the conductivity measurements: $\mathrm{w} \mathrm{mm}$ for the living sample and $4.5 \mathrm{~mm}$ for the dead one. The sample had to be shortened to just above $10 \mathrm{~cm}$ of length to have them straight to avoid movement of the sample outside the axis of rotation of the microtomography equipment. After the initial full scan, the samples were put in contact with approximatelys $\mathrm{ml}$ of water at the base of the vertically held twigs (i.e., no positive pressure). Low resolution scans were performed every $20 \mathrm{~min}$ for three hours. Four hours later,É second full scan was performed. The X-ray microtomography scans were conducted according to Suuronen et al. (2013). Briefly, desktop microtomography equipment (Nanotom $180 \mathrm{NF}$, Phoenix|x-ray Systems and Services, Wunstorf, Germany; currently owned by GE Measurement and Control Solutions) was used. One full scan was taken over É full $360^{\circ}$ rotation of the sample. Image reconstructions were performed with datos $\mid \mathrm{x}$-reconstruction software, provided by Phoenix|x-ray Systems and Services. Xylem embolised and refilled areas were calculated with ImagePro Plus (Media Cybernetics, Inc., Bethesda, MD, USA) based on the colour difference.

\section{RESULTS}

\section{Refilling under mild positive pressure}

The hydraulic conductivity recovered in living samples but not in killed ones (Figure 1), indicating that embolism refilled in living samples, and that living cells were important in 
facilitating refilling. On average, the first drop of water came through the living samples 87 min after they were placed in contact with water (Figure 1). The hydraulic conductance increased until 300 min after the branch was put in contact with water, then it remained more or less steady until the measurement was stopped at 700 min after contact with water. No water came through the killed samples for the $700 \mathrm{~min}$ that the experiment lasted. In all these experiments, the osmolality of the xylem sap extracted was $0.0391^{\prime \prime} 0.010 \mathrm{osmo} / \mathrm{kg}$.

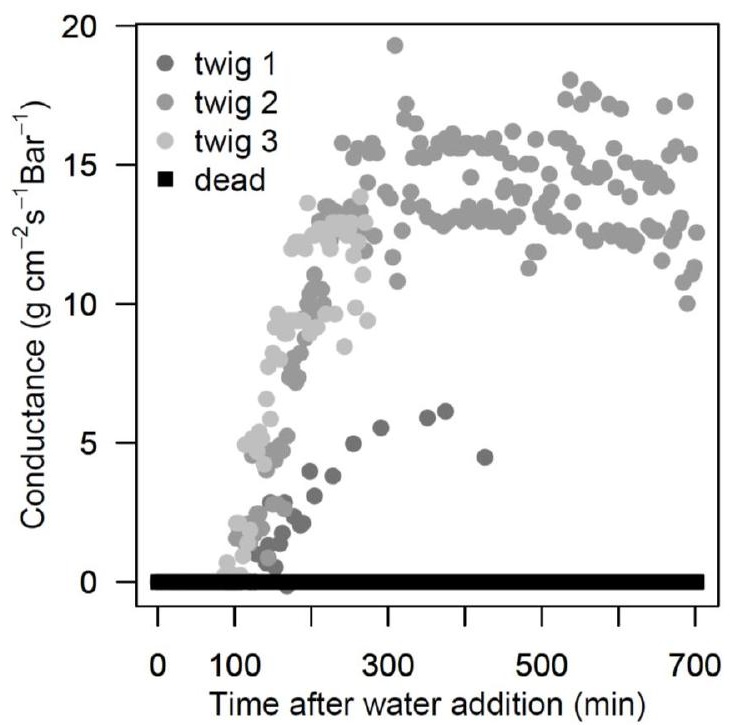

Figure 1. Hydraulic conductance of living (grey circles) and dead (black squares however, it showsE line at $\mathrm{y}=0)$ birch branches after being placed in contact with water $(\mathrm{n}=3)$.

\section{Refilling in agar}

After being in contact with the agar overnight, water potential of three twigs were measured at $0.0 \mathrm{MPa}$ (the top cut surface was wet), which is above the water potential (-0.1 $\mathrm{MPa}$ ) of the agar matrix used asÉ source for water. When the refilled twig was put in contact with water in É traditional hydraulic conductance measurement setup (see above), it immediately started to conduct water (Figure 2). The osmolality of the xylem sap extracted was $0.055^{\prime} 0.002 \mathrm{osmo} / \mathrm{kg}$.

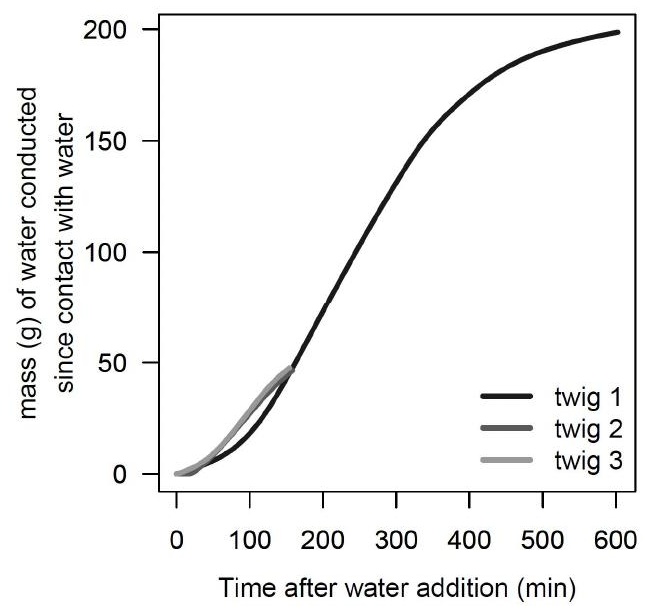

Figure 2: Mass of water flowing through living branches refilled in agar (at -0.1 MPa) through time. Timer indicates the time the branches are placed in contact with water. 


